\title{
Prevalence, Treatment, and Control of Hypercholesterolemia in High Cardiovascular Risk Patients: Evidences from a Systematic Literature Review in Spain
}

Alex de la Sierra · Xavier Pintó · Carlos Guijarro · José López Miranda •

Daniel Callejo · Jesús Cuervo · Rudi Subirà · Marta Rubio

To view enhanced content go to www.advancesintherapy.com Received: August 1, 2015 / Published online: October 26, 2015

(c) The Author(s) 2015. This article is published with open access at Springerlink.com

\begin{abstract}
Introduction: Cardiovascular diseases (CVDs) represent a major Public Health burden. High serum cholesterol levels have been linked to major CV risk. The objectives of this study were to review the epidemiology of hypercholesterolemia in high risk $\mathrm{CV}$ patients from Spain, by assessing its prevalence, the proportion of diagnosed patients undergoing
\end{abstract}

Electronic supplementary material The online version of this article (doi:10.1007/s12325-015-0252-y) contains supplementary material, which is available to authorized users.

A. de la Sierra $(\bowtie)$

Department of Internal Medicine, University

Hospital Mutua Terrassa, Barcelona, Spain

e-mail: adelasierra@mutuaterrassa.cat;

asierra@ub.edu

X. Pintó

Lipid Unit, Internal Medicine Service, University

Hospital of Bellvitge, Barcelona, Spain

C. Guijarro

Internal Medicine Service, University Hospital

Alcorcón Foundation, Madrid, Spain

J. L. Miranda

Lipids and Atherosclerosis Unit, IMIBIC/Reina Sofıa

University Hospital, University of Cordoba and

CIBER Fisiopatologia Obesidad y Nutricion,

Instituto de Salud Carlos III, Cordoba, Spain pharmacological treatment and the degree of attained lipid control.

Methods: A systematic literature review was carried out using Medline and two Spanish databases. Manuscripts containing information on hypercholesterolemia in several high CV risk groups [diabetes mellitus (DM), Systematic COronary Risk Evaluation (SCORE) risk $>5$, or documented CVD], published between January 2010 and October 2014, were included.

Results: Of the 1947 published references initially retrieved, a full-text review was done on 264 manuscripts and 120 were finally

\author{
J. L. Miranda \\ Reina Sofia University Hospital, IMIBIC/Fundacion \\ para la Investigacion Biomedica de Cordoba, \\ Cordoba, Spain \\ D. Callejo $\cdot$ J. Cuervo \\ BAP Health Outcomes Research, Oviedo, Spain \\ R. Subirà · M. Rubio \\ Health Economics and Outcomes Research, Sanofi \\ Iberia, Barcelona, Spain
}


included. Prevalence of hypercholesterolemia ranged from 50 to $84 \%$ in diabetics, $30-60 \%$ in patients with DM or elevated SCORE risk, $64-74 \%$ with coronary heart disease, 40-70\% in stroke patients, and $60-80 \%$ in those with peripheral artery disease. Despite the finding that most of them were on pharmacological treatment, acceptable control of serum lipids was very variable, ranging from $15 \%$ to $65 \%$. Among those with heterozygous familial hypercholesterolemia, $\quad 95-100 \%$ received treatment but less than 50\% achieved their therapeutic goals.

Conclusions: An elevated prevalence of hypercholesterolemia can be found in targeted groups at high CV risk. Although most patients are receiving pharmacological treatment, rates of lipid control continue to be low, both in primary and secondary prevention.

Keywords: Cardiovascular disease; Control; Drug; Dyslipidemia; Hypercholesterolemia; Prevalence

\section{INTRODUCTION}

The overall prevalence of hypercholesterolemia in the Spanish adult population has been estimated between $34 \%$ and $50 \%$ in recent studies [1-3]. The importance of this high prevalence is due to the association of high levels of serum cholesterol with cardiovascular disease (CVD), which has been well established $[4,5]$. For instance, the occurrence of CV events in patients with familial hypercholesterolemia has been highlighted, with a prevalence of premature CVD (before 55 years of age in men and before 65 years of age in women) of $10 \%$, in contrast to less than $3 \%$ in relatives without familial hypercholesterolemia [6].

The therapeutic arsenal available for hypercholesterolemia includes the standard treatment based on lifestyle and dietary modification strategies, and lipid-lowering medications, mainly statins [7]. Other existing treatments for controlling low-density lipoprotein cholesterol (LDLc) levels are ezetimibe (added to statins in primary hypercholesterolemia and homozygous familial hypercholesterolemia or as a monotherapy in cases of statin intolerance or if they are contraindicated), fibrates and bile-acid sequestrants. In addition, newly developed lipid-lowering drugs (monoclonal antibodies proprotein convertase subtilisin/ kexin type 9 inhibitor) have been recently approved by the Food and Drug Administration and European Medicines Agency. Lipid-lowering treatments (LLT) are efficacious in reducing LDLc levels and reducing the burden of major $\mathrm{CV}$ events as well as CV mortality, even among populations without known CVD [8, 9]. Consequently, recent European Clinical Practice Guidelines have established LDLc targets accordingly to individual's CV risk [7]: LDLc levels $<100 \mathrm{mg} / \mathrm{dl}$ for patients without CVD, but with diabetes mellitus (DM) or with high CV risk based on a Systematic COronary Risk Evaluation (SCORE) risk assessment $>5$ (Primary prevention hereinafter), and $<70 \mathrm{mg} / \mathrm{dl}$ for patients with overt CVD, i.e., those who have suffered a previous $\mathrm{CV}$ event (Secondary prevention). However, according to the results published in a multinational study conducted between 2006 and 2007, despite the use of LLT, a significant proportion of patients did not attain target levels of LDLc [10], which means they still have a significant $\mathrm{CV}$ residual risk. Understanding the contemporary magnitude of this condition is of importance to plan additional interventions to reach LDLc targets and to reduce the burden of the related $\mathrm{CV}$ events and deaths. Thus, the objectives of the 
present research were to review the published evidence addressing the prevalence of hypercholesterolemia, the usual clinical management of lipid profile, and the attained control of LDLc levels in patients with high CV risk from Spain.

\section{METHODS}

A systematic review of the literature was conducted in accordance with the accepted standards [11] and with a research question defined with reference to Patients, Interventions, Comparisons, Outcomes, and Study design (PICOS) (please see Table S1 in the supplementary material for details). The search was run on the second week of October 2014 (from 6th to 10th) in Medline and two Spanish electronic databases: Biblioteca Virtual de la Salud (BVS) and Medes. The search strategies included both controlled and free terms in both English and Spanish languages. The search strategies executed in each electronic public-access libraries are presented in Table S2 in the supplementary material.

The information gathered was manually cross-checked using the relevant references given in the publications included. To select the studies, titles and abstracts were first examined. After this, full text of selected manuscripts was reviewed. The selection criteria for publications were as follows:

\section{Inclusion Criteria}

Studies carried out in Spain and focused on lipid levels of patients belonging to one of the following groups: (a) heterozygous familial hypercholesterolemia (HeFH); (b) overt CVD including acute coronary syndrome (ACS), chronic coronary heart disease (CHD), ischemic stroke (IS) and peripheral arterial disease (PAD); (c) DM; or (d) high CV risk. ACS was defined as acute myocardial infarction or unstable angina in the last 12 months. Chronic CHD refers to patients with ACS occurred longer than 12 months, or with stable angina or revascularization. Regarding CV risk, a SCORE risk assessment $>5$ (tables for countries with low $\mathrm{CV}$ risk) or equivalent was considered. Furthermore, according to the objectives described before, the studies had to include information on one of the following results: (a) prevalence of hypercholesterolemia;

(b) rates of pharmacological treatment or (c) control of LDLc levels.

\section{Exclusion Criteria}

Manuscripts were discarded if any of the following conditions applied: (a) carried out outside Spain; (b) studies that did not include original patient data (i.e., reviews, editorials or letters); (c) studies that did not include human data; (d) studies with pediatric populations or (e) case reports.

\section{Compliance with Ethics Guidelines}

This article is based on previously conducted studies, and does not involve any new studies of human or animal subjects performed by any of the authors.

\section{RESULTS}

A total of 1362 publications were retrieved from PubMed, 537 from the BVS and 560 from Medes (512 were duplicates so an initial pool of 1947 original manuscripts were identified for title and abstract review). Following the selection process, the full text of 264 manuscripts was reviewed, of which a total of 120 original papers were finally considered (Fig. 1). 


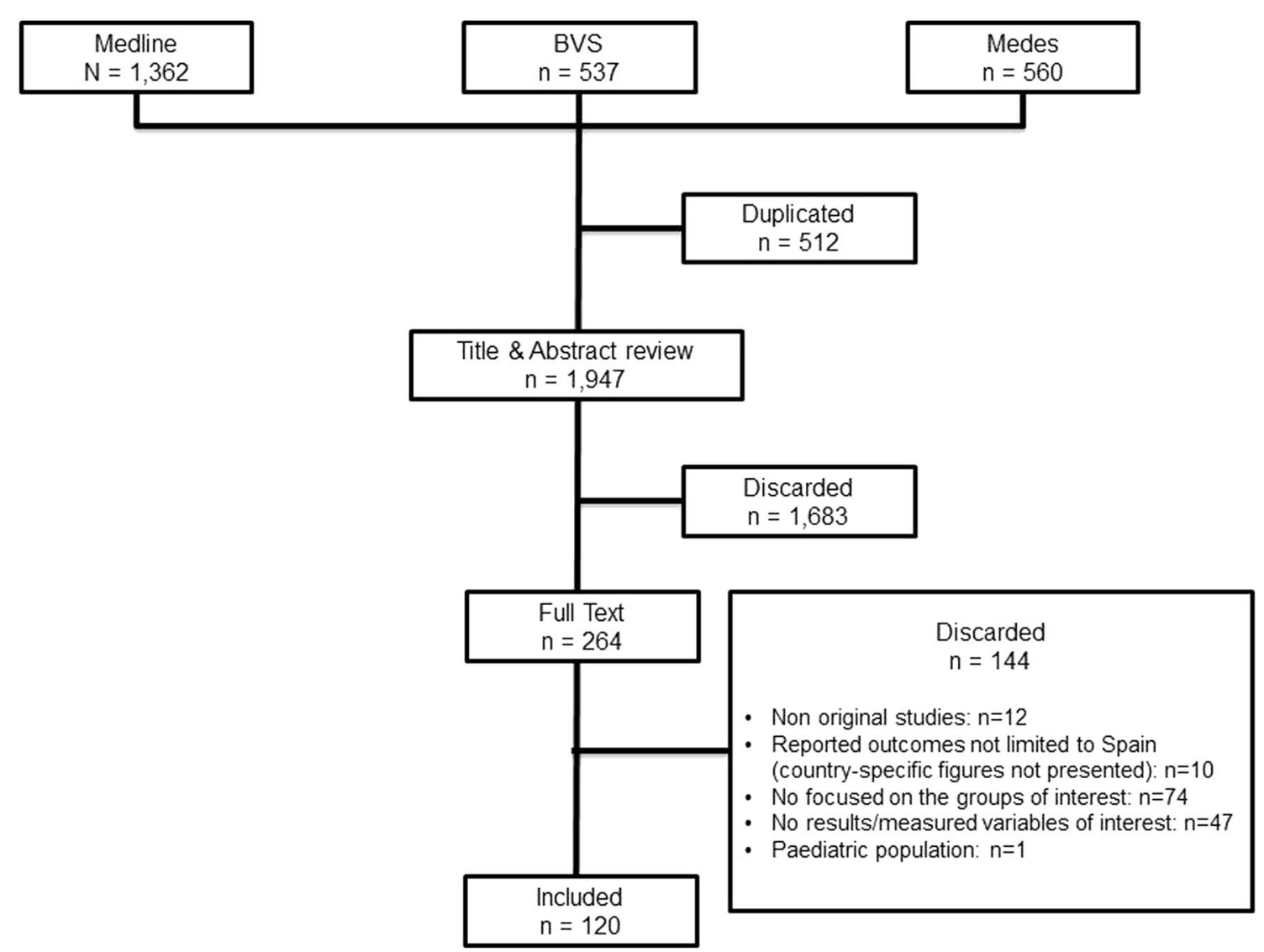

Fig. 1 Flow diagram showing the selection process of the included articles. BVS Biblioteca Virtual de la Salud

\section{HeFH}

In the study using data from the electronic database of the Spanish Familial Hypercholesterolemia Longitudinal Cohort Study (SAFEHEART) [12], 84\% of the HeFH patients were receiving LLT at the time of inclusion (97\% index cases and $78 \% \mathrm{HeFH}$ relatives). $95 \%$ of treated patients received statins, $58 \%$ as monotherapy and $31 \%$ in combination, mostly with ezetimibe. Meanwhile, in another observational study [13] which included 241 patients with $\mathrm{HeFH}$ and 286 with combined familial hypercholesterolemia, $100 \%$ received LLT during a monitoring period of 1 year. Patients took an average of 1.5 lipid-lowering drugs (94\% statins and 33\% ezetimibe).
Despite the high proportion of patients undergoing a LLT in both studies [12, 13], between $72 \%$ and $96 \%$, approximately, did not reach a target LDLc below $100 \mathrm{mg} / \mathrm{dl}$ and only $45 \%$ reduced LDLc levels by $>50 \%$ (Table 1 ).

\section{Hypercholesterolemia in Secondary Prevention}

\section{ACS}

In studies carried out among patients with ACS the prevalence of hypercholesterolemia was highly variable ranging from $45 \%$ to $80 \%$ [15-31]. The proportion of ACS patients undergoing LLT ranged between 33\% and $90 \%$, but control levels were poor with only $14 \%$ of patients with LDLc levels $<70 \mathrm{mg} / \mathrm{dl}$ and 
Table 1 Lipid-lowering treatments and LDL cholesterol targets in Heterozygous familial hypercholesterolemia

\begin{tabular}{|c|c|c|c|}
\hline Study & Study size & Treatment & Control \\
\hline$[12]^{a}$ & $\begin{array}{l}N=1852(1262 \mathrm{FH} \text { and } 590 \\
\text { relatives non } \mathrm{FH})\end{array}$ & $\begin{array}{l}\text { LLT: } 83.7 \%(97 \% \mathrm{FH} \text { and } 78 \% \\
\quad \text { relatives } \mathrm{FH}) \\
\text { Statins monotherapy: } 58.3 \% \\
\text { Statin + ezetimibe: } 31.3 \%\end{array}$ & $\begin{array}{l}\mathrm{LDLc}<100 \mathrm{mg} / \mathrm{dl}: 33(3.4 \%) \text { of } \\
\text { FH on LLT }\end{array}$ \\
\hline$[13]^{\mathrm{b}}$ & $\begin{array}{l}N=527(241 \mathrm{HeFH} \text { and } 286 \\
\text { combined } \mathrm{FH})\end{array}$ & $\begin{array}{l}\text { LLT: } 100 \% 1 \text { year after study entry } \\
\text { Statins: } 94.3 \% \\
\text { Ezetimibe: } 33.4 \%\end{array}$ & $\mathrm{LDLc}<100 \mathrm{mg} / \mathrm{dl}: 28.5 \%$ \\
\hline [14] & $37 \mathrm{HeFH}$ and 37 controls & $\begin{array}{l}\text { Statins: } 100 \% \mathrm{HeFH} \text { and } 100 \% \\
\text { no-HeFH } \\
\text { Ezetimibe: } 23(62.2 \%) \mathrm{HeFH} ; 4 \\
\quad(10.8 \%) \text { no-HeFH }\end{array}$ & LDLc $<100 \mathrm{mg} / \mathrm{dl}: 11 \%$ of $\mathrm{HeFH}$ \\
\hline
\end{tabular}

LLT lipid-lowering treatment, $F H$ familiar hypercholesterolemia, $L D L c$ low-density lipoprotein cholesterol, $H e F H$ heterozygous familial hypercholesterolemia

${ }^{a}$ Only $13 \%$ received maximum daily statin doses, defined as simvastatin $80 \mathrm{mg}$, pravastatin $40 \mathrm{mg}$, lovastatin $80 \mathrm{mg}$, fluvastatin $80 \mathrm{mg}$, atorvastatin $80 \mathrm{mg}$, rosuvastatin $20-40 \mathrm{mg}$ or maximum statin dose plus ezetimibe $10 \mathrm{mg} / \mathrm{day}$

b The strength of the lipid-lowering treatment in $\mathrm{HeFH}$ patients was considered low (lovastatin 10-40 mg, fluvastatin $80 \mathrm{mg}$, pravastatin $20-40 \mathrm{mg}$, simvastatin $10-20 \mathrm{mg}$, atorvastatin $10 \mathrm{mg}$ ) in $6.9 \%$ of cases, moderate (lovastatin $80 \mathrm{mg}$, simvastatin $40 \mathrm{mg}$, atorvastatin $20-40 \mathrm{mg}$, rosuvastatin $5-10 \mathrm{mg}$, simvastatin + ezetimibe $20+10 \mathrm{mg}$ ) in $40 \%$ and high (atorvastatin $80 \mathrm{mg}$, rosuvastatin $20 \mathrm{mg}$, simvastatin + ezetimibe $40+10 \mathrm{mg}$ ) in $53 \%$

$56 \%$ meeting the target of LDLc $<100 \mathrm{mg} / \mathrm{dl}$ [26] (Table 2; Fig. 2).

\section{CHD}

The prevalence of hypercholesterolemia in patients with chronic CHD was more uniform than that found in patients with ACS, according to the information collected, ranging from $64 \%$ to $74 \%[32-38,44-46]$. More than $80 \%$ of patients were receiving LLT, statins accounting for $95 \%$ of them [37]. Again, despite the high proportion of treatment, only between $26 \%$ and $55 \%$ of patients had LDLc levels $<100 \mathrm{mg} / \mathrm{dl}$ (Table 2).

\section{IS}

The prevalence of hypercholesterolemia in patients with IS ranged from $40 \%$ to $70 \%$
[39-41, 47-60]. The proportion of treated patients was lower than that found in ACS or CHD patients. Upon discharge from hospital following an IS episode, between 38\% and 76\% of patients were receiving LLT [39, 40, 49]. In addition, one observational study with 203 patients admitted for medium- to long-term stays in one hospital between 2009 and 2010 found that only $20 \%$ of the total number of post-event patients received the dosage recommended by current guidelines [54].

With regards to attained cholesterol levels, 3 studies were found [39-41], reporting figures on LDLc control of $<100 \mathrm{mg} / \mathrm{dl}$ between $25 \%$ and $33 \%$. In two additional studies $[54,61]$ in which targets were set at Total Cholesterol (TC) $<175 \mathrm{mg} / \mathrm{dl}$, control rates were between $43 \%$ and $77 \%$ (Table 2; Fig. 2). 
Table 2 Lipid-lowering treatments and LDL cholesterol targets in secondary prevention

\begin{tabular}{|c|c|c|c|}
\hline Study & Study size & Treatment & Control \\
\hline \multicolumn{4}{|c|}{ Acute coronary syndrome (ACS) } \\
\hline \multirow[t]{2}{*}[26]{} & $N=4334$ & Statins: $90.8 \%$ & LDLc $<70 \mathrm{mg} / \mathrm{dl}: 14.3 \%$ \\
\hline & & Statins + ezetimibe: $24.7 \%$ & LDLc $<100 \mathrm{mg} / \mathrm{dl}: 55.7 \%$ \\
\hline \multirow[t]{2}{*}[28]{} & $N=1381$ & & $\begin{array}{l}\text { LDLc }<70 \mathrm{mg} / \mathrm{dl}: 11 \% \text { first ACS; } 14.1 \% \\
\quad \text { recurrent ACS }\end{array}$ \\
\hline & & & $\begin{array}{l}\text { LDLc } 70-99 \mathrm{mg} / \mathrm{dl}: 24.1 \% \text { first ACS; } \\
23.2 \% \text { recurrent ACS }\end{array}$ \\
\hline \multicolumn{4}{|c|}{ Coronary heart disease (CHD) } \\
\hline$[32]$ & $N=7600$ & Statins: $80.6 \%$ & LDLc $<100 \mathrm{mg} / \mathrm{dl}: 26.1 \%$ \\
\hline \multirow[t]{4}{*}[33]{} & $N=1452(5256$ visits $)$ & Statins: $92.1 \%$ & LDLc $<70$ mg/dl: $292(5.7 \%)$ \\
\hline & & & LDLc $70-100 \mathrm{mg} / \mathrm{dl}$ (non DM): $916(18 \%)$ \\
\hline & & & LDLc $70-100 \mathrm{mg} / \mathrm{dl}(\mathrm{DM}): 640(12.6 \%)$ \\
\hline & & & LDLc $>100$ mg/dl: $3244(63.7 \%)$ \\
\hline [34] & $N=1108$ & $\begin{array}{l}\text { Statins: } 967 \text { (87.3\%); non DM: } \\
678 \text { (85.8\%); DM } 289 \text { (90.9\%) }\end{array}$ & $\begin{array}{l}\text { LDLc < } 100 \mathrm{mg} / \mathrm{dl}: 454 \text { (41\%); non DM: } \\
301 \text { (38.1\%); DM: } 153 \text { (48.1\%) }\end{array}$ \\
\hline$[35]$ & $N=7823$ & Statins: $80.4 \%$ & LDLc $>100 \mathrm{mg} / \mathrm{dl}: 73.8 \%$ \\
\hline \multirow[t]{2}{*}[36]{} & $N=1038$ & $\begin{array}{l}\text { Statins: } 82.9 \% ; 82.8 \%>65 \text { years; } \\
\quad 83.1 \% \leq 65 \text { years }\end{array}$ & $\begin{array}{l}\text { LDLc }<100: 42.4 \%>65 \text { years; } 46.5 \% \\
\quad \leq 65 \text { years }\end{array}$ \\
\hline & & $\begin{array}{l}\text { Ezetimibe } 17.4 \% ; 16.2 \% \\
\quad>65 \text { years; } 18.7 \% \leq 65 \text { years }\end{array}$ & \\
\hline \multirow[t]{3}{*}[37]{} & $N=2292$ & Statins: $94.1 \%$ & LDLc $>100 \mathrm{mg} / \mathrm{dl}: 44.9 \%$ \\
\hline & & Statins monotherapy: $74 \%$ & \\
\hline & & Ezetimibe: $18.3 \%$ & \\
\hline$[38]$ & $N=2024$ & & $\begin{array}{l}\text { LDLc }<100 \mathrm{mg} / \mathrm{dl}-\text { BMI 20-24.9: 35.2\%; } \\
\text { BMI 25-29.9: 30.5\%; BMI } \geq 30: 27.9 \% \text {. }\end{array}$ \\
\hline \multicolumn{4}{|c|}{ Ischemic stroke } \\
\hline \multirow[t]{2}{*}[39]{} & $N=473$ & LLT: $319(67.4 \%)$ & LDLc $<100 \mathrm{mg} / \mathrm{dl}: 33 \%$ \\
\hline & & Statins: $311(65.8 \%)$ & \\
\hline \multirow[t]{2}{*}[40]{} & $N=955$ & $\begin{array}{l}\text { LLT: } 75.5 \% \\
\text { Statins: } 695(72.8 \%)\end{array}$ & $\begin{array}{l}\mathrm{LDLc}<100 \mathrm{mg} / \mathrm{dl}: 28.9 \% \text { of treated } \\
\text { patients }\end{array}$ \\
\hline & & Ezetimibe: $76(8 \%)$ & \\
\hline$[41]$ & $N=407$ & $\begin{array}{l}\text { LLT: } 193(47.4 \%): \\
\text { Statins: } 180(44.2 \%)\end{array}$ & $\begin{array}{l}\text { LDLc }<100 \mathrm{mg} / \mathrm{dl}: 101(24.8 \%) ; \text { LDLc } \\
>100 \mathrm{mg} / \mathrm{dl}: 139 \text { (34.2\%); unknown: } \\
167(41.0 \%)\end{array}$ \\
\hline
\end{tabular}


Table 2 continued

\begin{tabular}{llll}
\hline Study & Study size & Treatment & Control \\
\hline Peripheral arterial disease (PAD) & & \\
{$[42]$} & $N=4087$ & LLT: $79.1 \%$ & LDLc $<100 \mathrm{mg} / \mathrm{dl}: 30.4 \%$ \\
& Statins: $76.2 \%$ & \\
{$[43]$} & $N=105$ & LLT: $45.7 \%$ & \\
\hline
\end{tabular}

LLT lipid-lowering treatment, $F H$ familiar hypercholesterolemia, $D M$ diabetes mellitus, $L D L c$ low-density lipoprotein cholesterol, $A C S$ acute coronary syndrome, $C H D$ coronary heart disease, BMI body mass index

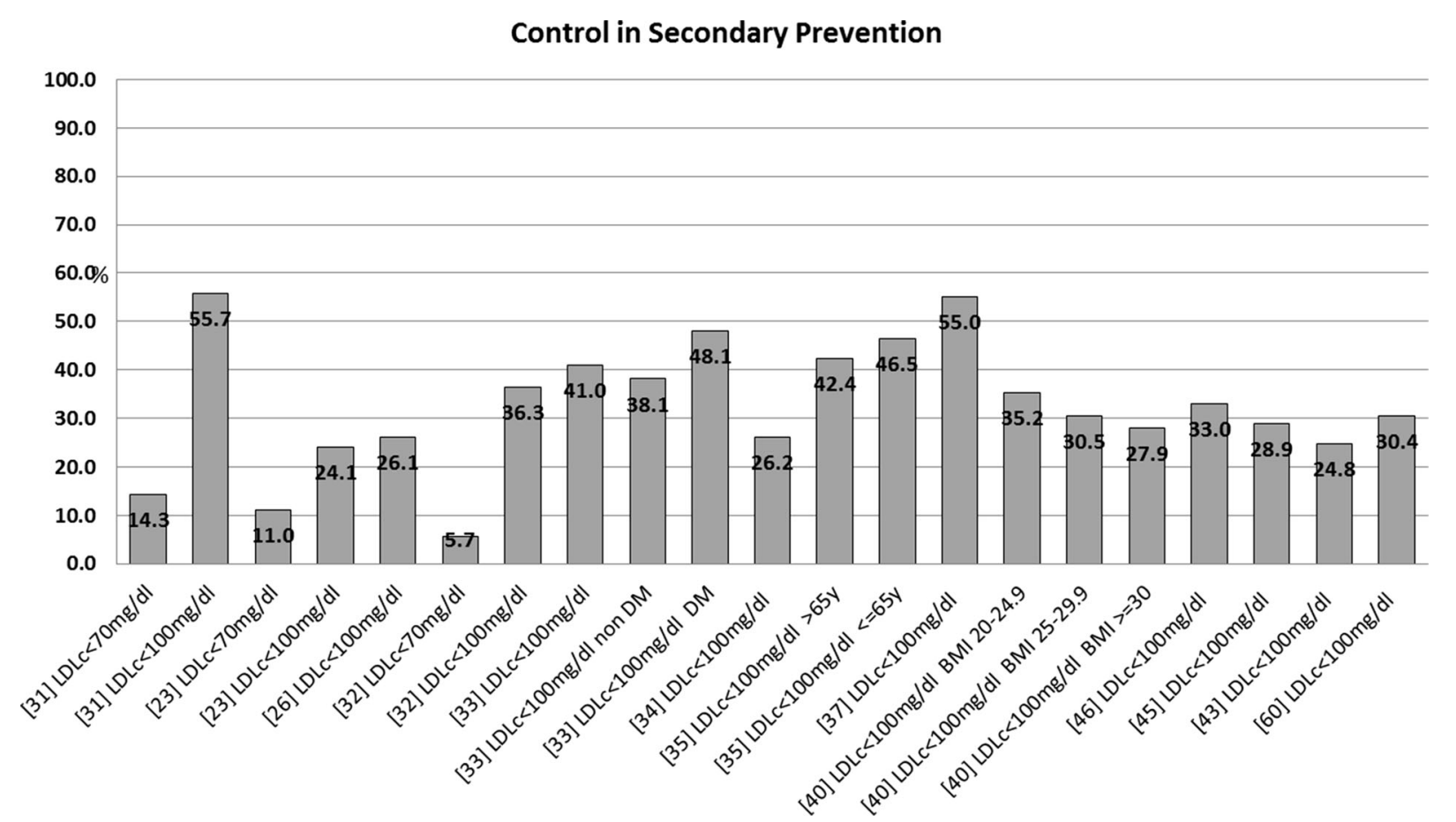

Fig. 2 Control in secondary prevention. $L D L c$ low-density lipoprotein cholesterol, $D M$ diabetes mellitus, $B M I$ body mass index

$P A D$

A very limited number of studies were identified for this particular subgroup [42, 62-64]. In these studies, the clinical diagnosis of PAD was based on an ankle-brachial index $<0.9$. A prevalence of hypercholesterolemia between $60 \%$ and $80 \%$ was reported. With regard to treatment applied and results achieved, between $46 \%$ and $79 \%$ of patients received LLT. However, only 30\% of them achieved a target LDLc $<100 \mathrm{mg} / \mathrm{dl}$ (Table 2; Fig. 2).

\section{Hypercholesterolemia in Primary Prevention}

\section{DM}

In accordance with a previous diagnosis or the use of LLT, data indicated that between 50\% 
Table 3 Lipid-lowering treatments and LDL cholesterol targets in primary prevention: diabetes mellitus

\begin{tabular}{|c|c|c|c|}
\hline Study & Study size & Treatment & Control \\
\hline$[33]$ & $\begin{array}{l}N=1452(612 \mathrm{DM}) \\
\text { patients; } 5256 \text { visits }\end{array}$ & Statins: $92.1 \%$ & $\begin{array}{l}\text { LDLc }<70 \mathrm{mg} / \mathrm{dl}: 292(5.7 \%) \\
\text { LDLc } 70-100 \mathrm{mg} / \mathrm{dl} \text { non DM: } 916 \\
\quad(18 \%) \\
\text { LDLc } 70-100 \mathrm{mg} / \mathrm{dl} \text { DM: } 640(12.6 \%) \\
\text { LDLc }>100 \mathrm{mg} / \mathrm{dl}: 3244(63.7 \%)\end{array}$ \\
\hline$[71]$ & $N=3703(1445 \mathrm{DM})$ & $\begin{array}{l}\text { Statins: } 100 \% \text { (during at least } \\
3 \text { months) }\end{array}$ & $\begin{array}{l}\text { LDLc }>100 \mathrm{mg} / \mathrm{dl}: 59.2 \% \text { of DM } \\
\text { patients } \\
\text { LDLc }>100 \mathrm{mg} / \mathrm{dl}: 44.5 \% \text { of non DM } \\
\text { patients }\end{array}$ \\
\hline$[72]$ & $\begin{array}{l}N=1828(320 \mathrm{DM}) . \\
2 \text { visits }\end{array}$ & $\begin{array}{l}\text { At baseline, } 55.4 \% \text { received } \geq 1 \\
\text { drug: } \\
\text { Statins: } 830(45.4 \%) \\
\text { Ezetimibe: } 126(6.9 \%) \\
\text { Statin + ezetimibe: } 8(0.4 \%) \\
\text { At follow up: } \\
\text { Statins: } 1232(67.4 \%) \\
\text { Ezetimibe: } 332(18.2 \%) \\
\text { Statin + ezetimibe: } 61(3.3 \%)\end{array}$ & $\begin{array}{l}\text { LDLc }<100 \mathrm{mg} / \mathrm{dl} \text { in } \mathrm{DM} \text { or CVD and } \\
\text { LDLc }<115 \text { in high risk patients: } \\
\text { Baseline: All } 30.5 \% \text {; CVD } 40.4 \% \text {; DM } \\
35.8 \% \\
\text { Follow up: All } 44.7 \% \text {; CVD } 65.3 \% \text {; DM } \\
50.4 \% \\
\text { LDLc }<70 \text { : CVD } 17.9 \% \text {; DM } 16.5 \%\end{array}$ \\
\hline$[26]$ & $N=4402(1748 \mathrm{DM})$ & $\begin{array}{l}\text { Statins: } 76.7 \% \text { : } \\
\text { Statins + ezetimibe: } 18.8 \%\end{array}$ & $\begin{array}{l}\mathrm{LDLc}>100 \mathrm{mg} / \mathrm{dl}: 56.9 \% \\
\mathrm{LDLc}>70 \mathrm{mg} / \mathrm{dl}: 84.7 \%\end{array}$ \\
\hline$[73]$ & $N=3710(39 \% \mathrm{DM})$ & $\begin{array}{l}\text { Statins: } 100 \% \\
\text { Ezetimibe: } 17.4 \%\end{array}$ & $\begin{array}{l}\text { LDLc }>100 \mathrm{mg} / \mathrm{dl} \text { in high risk or } \\
\quad>120 \mathrm{mg} / \mathrm{dl} \text { in low risk: } 63.1 \% \\
\text { CVD }(n=846) ; \mathrm{LDLc}>100 \mathrm{mg} / \mathrm{dl}: \\
64.7 \%\end{array}$ \\
\hline$[3]$ & $\begin{array}{l}N=11,544 \\
\text { Rate of awareness: } 53.6 \% \\
\quad(53.5 \% \text { males; } 53.7 \% \\
\text { women) }\end{array}$ & $\begin{array}{l}\text { LLT treatment: } \\
44.1 \% \text { of patients aware of } \\
\text { elevated LDLc } \\
23.7 \% \text { of all patients with } \\
\text { elevated LDLc }\end{array}$ & $\begin{array}{l}\text { LDLc }<115 \mathrm{mg} / \mathrm{dl}(<100 \mathrm{mg} / \mathrm{dl} \mathrm{DM} \\
\text { and CVD): } 40.2 \% \text { of treated patients } \\
(9.5 \% \text { of total sample with elevated } \\
\text { LDLc) } \\
\text { LDLc }<115 \mathrm{mg} / \mathrm{dl}(<70 \mathrm{DM} \text { and } \\
\text { CVD): } 31.3 \%(7.3 \%) \\
\% \text { of DM or CVD patients with LDLc } \\
<100 \mathrm{mg} / \mathrm{dl}: 40.5 \% \text { or } 43.6 \% \text {, respect } \\
\% \text { of DM or CVD patients with LDLc } \\
<70 \mathrm{mg} / \mathrm{dl}: 7.0 \% \text { or } 5.2 \% \text { respectively }\end{array}$ \\
\hline
\end{tabular}


Table 3 continued

\begin{tabular}{|c|c|c|c|}
\hline Study & Study size & Treatment & Control \\
\hline \multirow[t]{6}{*}[67]{} & $N=2412$ & Before clinical session: & Before clinical session: \\
\hline & & Statins: $59.5 \%$ & LDLc $<100$ mg/dl: $22.7 \%$ \\
\hline & & Ezetimibe: $0.9 \%$ & At clinical session: \\
\hline & & At clinical session: & LDLc $<100$ mg/dl: $28.6 \%$ \\
\hline & & Statins: $65.5 \%$ & \\
\hline & & Ezetimibe: $4.2 \%$ & \\
\hline$[68]$ & $N=1177$ & Statins $48 \%$ & LDLc $<100 \mathrm{mg} / \mathrm{dl}: 25.6 \%$ \\
\hline \multirow[t]{3}{*}[74]{} & $N=4776(12.5 \% \mathrm{DM}$ & & In $n=409 \mathrm{DM}$ patients \\
\hline & patients) & & $\mathrm{LDL} \leq 100 \mathrm{mg} / \mathrm{dl}: 45.3 \%$ \\
\hline & & & $\mathrm{LDL} \leq 70 \mathrm{mg} / \mathrm{dl}: 11.8 \%$ \\
\hline \multirow[t]{2}{*}[66]{} & $N=771 \mathrm{DM}$ & Statin: $722(93.6 \%)$ & LDLc $>70 \mathrm{mg} / \mathrm{dl}: 501(73.4 \%)$ \\
\hline & & Ezetimibe: $151(19.6 \%)$ & LDLc $>100 \mathrm{mg} / \mathrm{dl}: 243(31.5 \%)$ \\
\hline \multirow[t]{2}{*}[75]{} & $N=2704(1067 \mathrm{DM})$ & LLT: 1634 (60.4\%) & $\begin{array}{l}\text { LDLc }<100 \mathrm{mg} / \mathrm{dl} \text { in } \mathrm{DM} \text { or CVD; } \\
\quad<130 \mathrm{mg} / \mathrm{dl} \text { others: } 930(34.4 \%)\end{array}$ \\
\hline & & & $\begin{array}{l}\text { LDLc }<100 \mathrm{mg} / \mathrm{dl}: 34.7 \% \mathrm{DM} \\
34.2 \% \mathrm{CVD}\end{array}$ \\
\hline \multirow[t]{4}{*}[76]{} & $N=1748 \mathrm{DM}$ and & LLT: $76.7 \%$ & LDLc $>100 \mathrm{mg} / \mathrm{dl}: 56.9 \%$ \\
\hline & CHD & Statin and ezetimibe: $18.8 \%$ & LDLc $>70 \mathrm{mg} / \mathrm{dl}: 84.7 \%$ \\
\hline & $N=2654 \mathrm{DM}$ without & & \\
\hline & CHD & & \\
\hline$[69]$ & $N=320 \mathrm{DM}$ & Statins: $60 \%$ & $\begin{array}{l}\text { LDLc } \leq 100 \mathrm{mg} / \mathrm{dl}: \text { males } 41.7 \% \text {; } \\
\text { females } 39.1 \%\end{array}$ \\
\hline
\end{tabular}

LLT lipid-lowering treatment, $F H$ familiar hypercholesterolemia, $D M$ diabetes mellitus, $C V D$ cardiovascular disease, $L D L c$ low-density lipoprotein cholesterol, $C H D$ coronary heart disease

and $84 \%$ of DM patients would present with hypercholesterolemia [44, 57, 65-68].

Regarding the rates of LLT usage (Table 3; Fig. 3), between $45 \%$ and $90 \%$ of DM patients were using a LLT $[26,69,70]$. With respect to the lipid control achieved in this population, rates ranged from $40 \%$ to $50 \%$ in DM patients with no CVD history (LDLc goal $<100 \mathrm{mg} / \mathrm{dl}$ ), to only $15 \%$ in DM patients with a history of CVD (LDLc goal $<70 \mathrm{mg} / \mathrm{dl}$ ).

\section{Patients with High CV Risk}

Within this group of special interest in the prevention of CVD, between $30 \%$ and $60 \%$ had a specific diagnosis of hypercholesterolemia, based on a TC $>200 \mathrm{mg} / \mathrm{dl}$ or previous treatment [1, 3, 76-78].

LLT was used in $50 \%$ to $60 \%$ of these patients [1], while control rates ranged from $35 \%$ to $65 \%$, considering a LDLc target of $<100 \mathrm{mg} / \mathrm{dl}[1,72$, 73]. Table 4 and Fig. 4 summarize these data. 


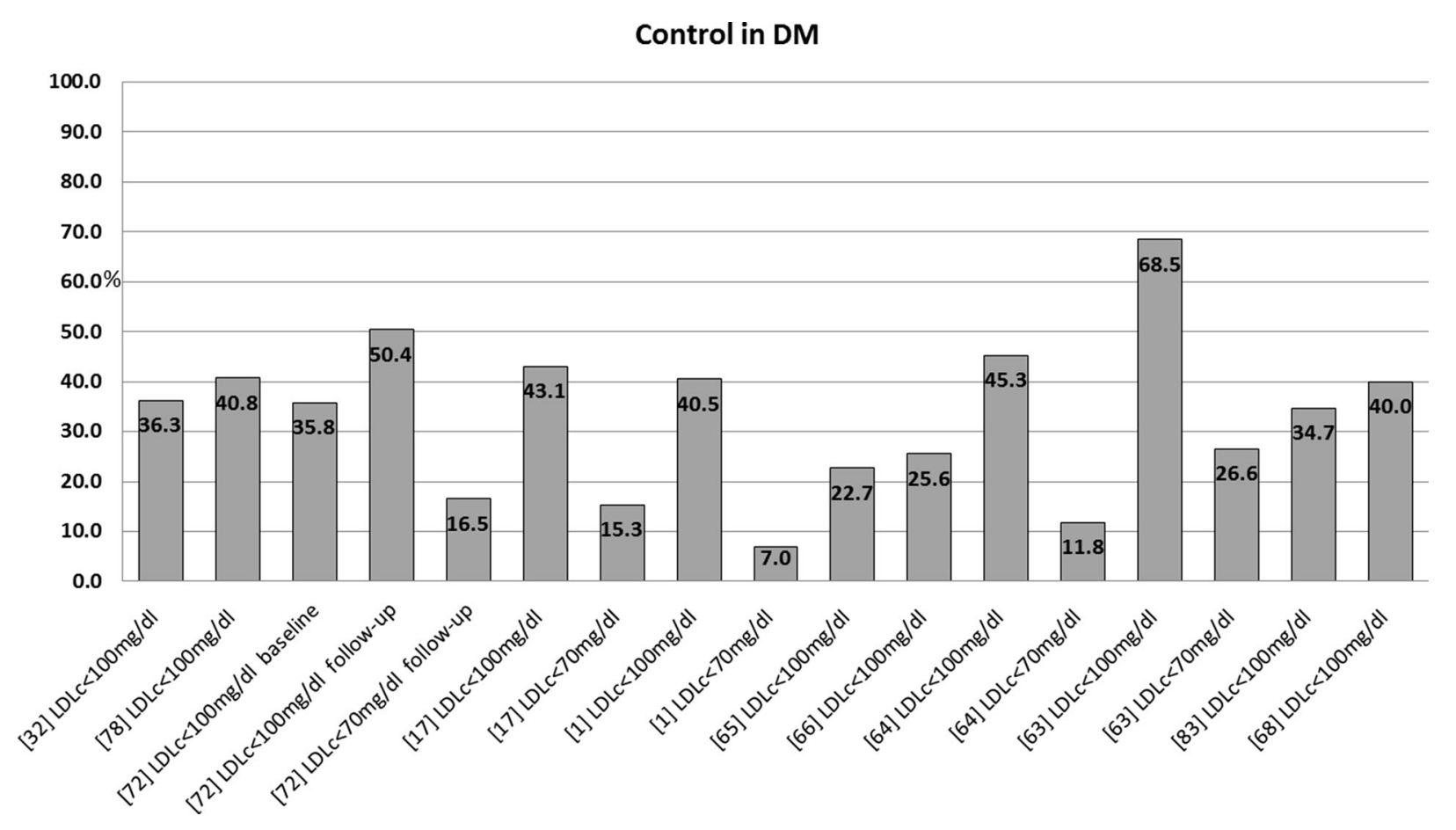

Fig. 3 Control in primary prevention: diabetes mellitus. $L D L c$ low-density lipoprotein cholesterol, $D M$ diabetes mellitus

\section{DISCUSSION}

It has been shown that hypercholesterolemia is significantly present among patients with an increased vascular risk and those who have already suffered a CV event: $50-84 \%$ of patients with DM $[44,57,67,68,74], 30-50 \%$ of high risk patient [1, 3, 76-78], and between $35 \%$ and above $80 \%$ in those with overt CVD, depending on the type of event [15-41, 44-60]. The association of this condition with the risk of CV events $[9,83]$ makes it all the more necessary to endorse the interventions aimed at managing the modifiable risk factors (diet and exercise), to prescribe a LLT accordingly to individual's CV risk [7], and also to plan an adequate monitoring of the pharmacological therapies implemented to maximize their benefit. There are many examples of this relationship in our country: hypercholesterolemia doubled the risk of an ischemic disease $[84,85]$, and would be the cause of $22 \%$ of all coronary events. The risk increased significantly among patients who did not have their lipid values controlled, while among those diagnosed with controlled hypercholesterolemia and, who are undergoing a lipid-lowering treatment, the increased risk of ischemic heart disease was not statistically significant [84].

Due, in large part, to the information above, the use of lipid-lowering drugs in Spain has increased from 18.9 defined daily doses (DDD) per 1000 inhabitants per day (DDD/1000 inhabitants/day) in the year 2000 to 102.6 DDD/1000 inhabitants/day in the year 2012, an increase of $442 \%$ [86]. Statins are the most commonly used drugs (91.7 DDD/1000 inhabitants/day in 2012) representing 89.3\% of all LLT, but also fibrates, bile-acid sequestrants, ezetimibe, and omega 3 fatty acids contributed during 2012 to LLT use [86]. The increase in the use of LLT can be justified by 
Table 4 Lipid-lowering treatments and LDL cholesterol targets in primary prevention: high cardiovascular risk patients

High or very high cardiovascular risk

\begin{tabular}{|c|c|c|c|}
\hline Study & Study size & Treatment & Control \\
\hline [73] & $N=3710(39 \% \mathrm{DM})$ & $\begin{array}{l}\text { Statins: } 100 \% \\
\text { Ezetimibe: } 17.4 \%\end{array}$ & $\begin{array}{l}\text { High risk patients (CVD, DM or SCORE } \\
\quad>5 \% ; n=2574), \mathrm{LDLc}>100 \mathrm{mg} / \mathrm{dl}: 60.7 \% \\
\text { SCORE }>5 \% \text { without CVD nor DM } \\
\quad(n=407), \mathrm{LDLc}>100 \mathrm{mg} / \mathrm{dl}: 83.2 \%\end{array}$ \\
\hline [72] & $N=18282$ visits & $\begin{array}{l}\text { At baseline, } 1013(55.4 \%) \\
\text { received at least } 1 \text { drug: } \\
\text { Statins: } 830(45.4 \%) \\
\text { Ezetimibe: } 126(6.9 \%) \\
\text { Statin + ezetimibe: } 8(0.4 \%) \\
\text { At follow up: } \\
\text { Statins: } 1232(67.4 \%) \\
\text { Ezetimibe: } 332(18.2 \%) \\
\text { Statin + ezetimibe: } 61(3.3 \%)\end{array}$ & $\begin{array}{l}\text { LDLc levels }<100 \mathrm{mg} / \mathrm{dl} \text { in } \mathrm{DM} \text { or CVD and } \\
\text { LDLc }<115 \text { in high risk patients: } \\
\text { Baseline: All } 30.5 \% \text {; CVD 40.4\%; DM } 35.8 \% \\
\text { Follow up: All 44.7\%; CVD 65.3\%; DM 50.4\% } \\
\text { LDLc }<70 \mathrm{mg} / \mathrm{dl}: \text { CVD 17.9\%; DM } 16.5 \%\end{array}$ \\
\hline [79] & $\begin{array}{l}\text { RCT. } 2 \text { arms: } \\
\text { Experimental-EG } \\
(n=33) \text { Supportive } \\
\text { system to decision } \\
\text { making } \\
\text { Control-CG }(n=44)\end{array}$ & $\begin{array}{l}\text { Use of High intensity statins: } \\
\text { EG: } 74.6 \% / C G: 25.4 \% \\
\text { Statins + ezetimibe or } \\
\text { niacin/laropiprant: } \\
\text { GI: } 32.4 \% / G C: 2.3 \%\end{array}$ & $\begin{array}{l}\text { After } 12 \text { weeks: } \\
\text { LDLc < } 70: 55 \% \text { GI; } 12.5 \% \text { GC } \\
\text { LDLc }<100: 75 \% \text { GI; } 45.8 \% \text { GC }\end{array}$ \\
\hline$[80]$ & $N=37$ & $\begin{array}{l}\text { Statins: } 100 \% \\
\text { Ezetimibe: } 10.8 \%\end{array}$ & LDLc $<130 \mathrm{md} / \mathrm{dl}: 43 \%$ \\
\hline [81] & $N=222$ & LLT: $85 \%$ & $\begin{array}{l}\text { LDLc }<100 \mathrm{mg} / \mathrm{dl}: 51.3 \% \text { of high risk patients } \\
\mathrm{LDLc}<70 \mathrm{mg} / \mathrm{dl}: 7.5 \% \text { of high risk patients }\end{array}$ \\
\hline$[77]$ & $\begin{array}{l}N=3716(15.5 \% \\
\text { calibrated-SCORE } \\
>5)\end{array}$ & Statins: $25.3 \%$ & $\begin{array}{l}\text { Patients with SCORE between } 5 \text { and } 10 \text { : } \\
\text { LDLc }<100 \mathrm{mg} / \mathrm{dl}: 10.61 \% \\
\text { SCORE }>10 \\
\text { LDLc }<70 \mathrm{mg} / \mathrm{dl}: 1.79 \%\end{array}$ \\
\hline [1] & $\begin{array}{l}N=27,903(n=9335 \\
\text { with dyslipidemia }) \\
\text { High-very high risk: } \\
11.3 \% \text { males and } 2.3 \% \\
\text { female }\end{array}$ & $\begin{array}{l}\text { LLT REGICOR >10: } 50 \% \\
\text { males, } 59 \% \text { females }\end{array}$ & $\begin{array}{l}\text { LDLc }<100 \mathrm{mg} / \mathrm{dl} \text { in DM or high-very high } \\
\text { risk: }<3 \% \\
\text { LDLc }<100 \mathrm{mg} / \mathrm{dl} \text { in } \mathrm{DM} \text { or }<130 \mathrm{mg} / \mathrm{dl} \text { in } \\
\text { moderate to very high risk or }<160 \mathrm{mg} / \mathrm{dl} \text { in } \\
\text { low risk): } 46 \% \text { males, } 52 \% \text { females }\end{array}$ \\
\hline
\end{tabular}


Table 4 continued

\begin{tabular}{|c|c|c|c|}
\hline \multicolumn{4}{|c|}{ High or very high cardiovascular risk } \\
\hline$\overline{\text { Study }}$ & Study size & Treatment & Control \\
\hline$[75]$ & $N=2704$ & LLT: $1634(60.4 \%)$ & $\begin{array}{l}\text { LDLc }<100 \mathrm{mg} / \mathrm{dl} \text { in DM or CVD; }<130 \mathrm{mg} / \\
\mathrm{dl} \text { others: } 930(34.4 \%) \\
\text { LDLc }<100 \mathrm{mg} / \mathrm{dl}: 34.7 \% \mathrm{DM} ; 34.2 \% \mathrm{CVD}\end{array}$ \\
\hline$[82]$ & $N=217$ & $\begin{array}{l}\text { Ezetimibe (monotherapy): } \\
\quad 42.4 \% \\
\text { Ezetimibe + statins: } 43.3 \%\end{array}$ & $\begin{array}{l}\mathrm{LDLc}<100 \mathrm{mg} / \mathrm{dl} \text { or } 70 \mathrm{mg} / \mathrm{dl}: 43.8 \% \text { of high } \\
\text { or very high risk patients }\end{array}$ \\
\hline$[3]$ & $\begin{array}{l}N=11,544 \\
\text { Rate of awareness: } 53.6 \% \\
\quad(53.5 \% \text { males; } 53.7 \% \\
\text { women })\end{array}$ & $\begin{array}{l}\text { LLT treatment: } \\
44.1 \% \text { of patients aware of } \\
\text { elevated LDLc } \\
23.7 \% \text { of all patients with } \\
\text { elevated LDLc }\end{array}$ & $\begin{array}{l}\text { LDLc }<115 \mathrm{mg} / \mathrm{dl}(<100 \mathrm{DM} \text { and CVD): } \\
40.2 \% \text { of treated patients }(9.5 \% \text { of total } \\
\text { sample with elevated LDLc) } \\
\text { LDLc }<115 \mathrm{mg} / \mathrm{dl}(<70 \mathrm{DM} \text { and CVD): } \\
31.3 \%(7.3 \%) \\
\text { \% of DM or CVD patients with } \\
\text { LDLc }<100 \mathrm{mg} / \mathrm{dl}: 40.5 \% \text { or } 43.6 \% \text {, respect. } \\
\% \text { of DM or CVD patients with LDLc } \\
<70 \mathrm{mg} / \mathrm{dl}: 7.0 \% \text { or } 5.2 \% \text { respectively }\end{array}$ \\
\hline
\end{tabular}

$L L T$ lipid-lowering treatment, $F H$ familiar hypercholesterolemia, $D M$ diabetes mellitus, $C V D$ cardiovascular disease, $L D L c$ low-density lipoprotein cholesterol, $E G$ experimental group, $C G$ control group

their ability to reduce the risk of CV events, and the associated burden of disease.

Although treatments available have led to an improvement in the clinical situation and prognosis of these cases, it is clear that there is a lot of effort still to be made until LDLc targets defined by current European guidelines [7] can be reached by most of patients. In all of the groups analyzed, an important number could still be found with total cholesterol and LDLc levels above the acceptable threshold. For example, among diabetics, only between 15\% (in case of those with previous CVD) and $40-50 \%$ (in those without CVD) attained recommended levels of lipids in the blood [26, 69-71], and the situation is not better in secondary prevention or among patients with HeFH. This picture, which is similar to other western countries [87-89], highlights the need to continue maximizing the control of lipids in the blood and to minimize therapeutic inertia. Regarding this, in one recent study with CHD patients, therapeutic inertia was estimated to be as high as 73\% [33].

It should be noted, as the main limitation of the present work, how difficult it is to make a more exact approximation of the estimations presented in this manuscript. This is due in great part to the sheer heterogeneity of the criteria used in the various studies in defining hypercholesterolemia, CV risk, ACS or CHD. In addition, one other relevant aspect to consider is the nature of the studies included. They are mainly cross-sectional investigations and with a wide range of different sampling procedures and sample sizes. Even more, many studies are based on the analysis of individuals coming from the same national databases or registries. 


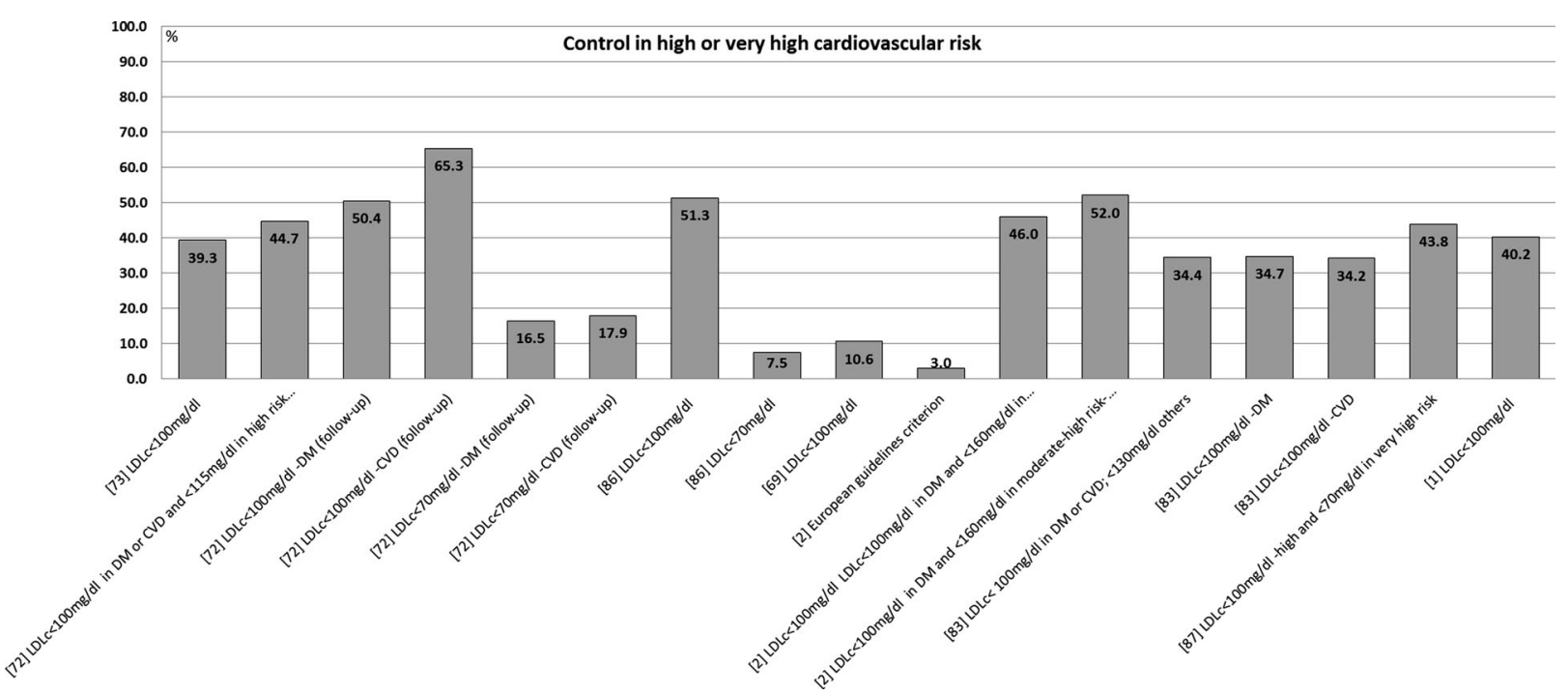

Fig. 4 Control in primary prevention: high or very high risk patients. $L D L c$ low-density lipoprotein cholesterol, $D M$ diabetes mellitus, $C V D$ cardiovascular disease

Considering these important constraints, a more analytic approach as meta-analysis might be applied at least with those studies following comparable clinical criteria to have a more accurate estimation of disease prevalence and control in each of the groups of interest, and to highlight the significance of the heterogeneity found between studies. Despite this, an important strength of the present manuscript is that all the relevant studies carried out in Spain and published in peer review journals have been systematically identify and reviewed to facilitate a global but comprehensive report of this condition in our country.

\section{CONCLUSIONS}

There is an elevated prevalence of hypercholesterolemia in Spain among those selected groups with a high CV risk. Although LLT is present in an elevated proportion, controls rates of lipid levels need to be improved both in primary and secondary prevention.

\section{ACKNOWLEDGMENTS}

Sponsorship, article processing charges, and the open access charge for this study were funded by Sanofi Iberia. All named authors meet the International Committee of Medical Journal Editors (ICMJE) criteria for authorship for this manuscript, take responsibility for the integrity of the work as a whole, and have given final approval to the version to be published.

Disclosures. Marta Rubio works at the Health Economics and Outcomes Research department of Sanofi Iberia. Rudi Subirà works at the Health Economics and Outcomes Research department of Sanofi Iberia. Alex de la Sierra has served as a scientific advisor to Sanofi and declares honoraria for participation in advisory board/scientific meetings funded by Abbott, Daiichi-Sankyo, Lacer, Menarini, Merck, Sharp and Dohme, Pfizer, and Sanofi. Xavier Pintó has served as a scientific advisor to Sanofi. Carlos Guijarro has served as a scientific advisor to Sanofi and declares honoraria as a speaker or consultant from Sanofi, MSD, Astra Zeneca. José 
López Miranda has served as a scientific advisor to Sanofi and declares fees for participation in advisory board/scientific meetings from Sanofi. Daniel Callejo has served as a scientific advisor to Sanofi. Jesús Cuervo has served as a scientific advisor to Sanofi.

Compliance with ethics guidelines. This article is based on previously conducted studies, and does not involve any new studies of human or animal subjects performed by any of the authors.

Open Access. This article is distributed under the terms of the Creative Commons Attribution-NonCommercial 4.0 International License (http://creativecommons.org/licenses/ by-nc/4.0/), which permits any noncommercial use, distribution, and reproduction in any medium, provided you give appropriate credit to the original author(s) and the source, provide a link to the Creative Commons license, and indicate if changes were made.

\section{REFERENCES}

1. Baena-Díez JM, Félix FJ, Grau M, Cabrera De León A, Sanz H, Leal M, et al. Tratamiento y control de los factores de riesgo según el riesgo coronario en la población española del estudio DARIOS. Rev Esp Cardiol. 2011;64:766-73.

2. Grau M, Elosua R, Cabrera De León A, Guembe MJ, Baena-Díez JM, Vega Alonso T, et al. Factores de riesgo cardiovascular en España en la primera década del siglo XXI: análisis agrupado con datos individuales de 11 estudios de base poblacional, estudio DARIOS. Rev Esp Cardiol. 2011;64:295-304.

3. Guallar-Castillón P, Gil-Montero M, León-Muñoz LM, Graciani A, Bayán-Bravo A, Taboada JM, et al. Magnitud y manejo de la hipercolesterolemia en la población adulta de España, 2008-2010: el estudio ENRICA. Rev Esp Cardiol. 2012;65:551-8.

4. Alzamora MT, Fores R, Baena-Diez JM, Pera G, Toran P, Sorribes $\mathrm{M}$, et al. The peripheral arterial disease study (PERART/ARTPER): prevalence and risk factors in the general population. BMC Public Health. 2010;10:38.

5. Ramos R, Balló E, Marrugat J, Elosua R, Sala J, Grau M, et al. Validez del Sistema de Información para el Desarrollo de la Investigación en Atención Primaria (SIDIAP) en el estudio de enfermedades vasculares: estudio EMMA. Validity for use in research on vascular diseases of the SIDIAP (Information System for the Development of Research in Primary Care): the EMMA study. Rev Esp Cardiol. 2012;65(1):29-37.

6. Alonso R, Andres E, Mata N, Fuentes-Jimenez F, Badimon L, Lopez-Miranda J, et al. Lipoprotein(a) levels in familial hypercholesterolemia: an important predictor of cardiovascular disease independent of the type of LDL receptor mutation. J Am Coll Cardiol. 2014;63(19):1982-9.

7. Perk J, De Backer G, Gohlke H, Graham I, Reiner Z, Verschuren $\mathrm{M}$, et al. European Guidelines on cardiovascular disease prevention in clinical practice (version 2012). The Fifth Joint Task Force of the European Society of Cardiology and Other Societies on Cardiovascular Disease Prevention in Clinical Practice (constituted by representatives of nine societies and by invited experts). Eur Heart J. 2012;33(13):1635-701.

8. Baigent C, Blackwell L, Emberson J, Holland LE, Reith $\mathrm{C}$, Bhala N, et al. Efficacy and safety of more intensive lowering of LDL cholesterol: a meta-analysis of data from 170,000 participants in 26 randomised trials. Lancet. 2010;376(9753):1670-81.

9. Mihaylova B, Emberson J, Blackwell L, Keech A, Simes J, Barnes EH, et al. The effects of lowering LDL cholesterol with statin therapy in people at low risk of vascular disease: meta-analysis of individual data from 27 randomised trials. Lancet. 2012;380(9841):581-90.

10. Waters DD, Brotons C, Chiang CW, Ferrieres J, Foody J, Jukema JW, et al. Lipid treatment assessment project 2: a multinational survey to evaluate the proportion of patients achieving low-density lipoprotein cholesterol goals. Circulation. 2009;120(1):28-34.

11. Perestelo-Pérez L. Standards on how to develop and report systematic reviews in Psychology and Health. Int J Clin Health Psychol. 2013;13:49-57.

12. Mata N, Alonso R, Badimon L, Padro T, Fuentes F, Muniz $O$, et al. Clinical characteristics and evaluation of LDL-cholesterol treatment of the Spanish Familial Hypercholesterolemia Longitudinal Cohort Study (SAFEHEART). Lipids Health Dis. 2011;10:94.

13. Lahoz C, Mostaza JM, Pinto X, de la Cruz JJ, Banegas JR, Pedro-Botet J. LDL-cholesterol control 
in patients with genetic dyslipidemia followed up by Lipid and Vascular Risk Units of the Spanish Society of Arteriosclerosis. Clin Investig Arterioscler. 2015;27(1):1-8.

14. Suades R, Padro T, Alonso R, Lopez-Miranda J, Mata $\mathrm{P}$, Badimon L. Circulating CD45+/CD3+ lymphocyte-derived microparticles map lipid-rich atherosclerotic plaques in familial hypercholesterolaemia patients. Thromb Haemost. 2014;111(1):111-21.

15. Aros F, Heras M, Vila J, Sanz $H$, Ferreira-Gonzalez I, Permanyer-Miralda G, et al. Reduction in 28 days and 6 months of acute myocardial infarction mortality from 1995 to 2005. Data from PRIAMHO I, II and MASCARA Registries. Rev Esp Cardiol (Engl Ed). 2011;64(11):972-80.

16. Cordero A, Moreno-Arribas J, Bertomeu-González V, Agudo P, Miralles B, Masiá $M$, et al. Las concentraciones bajas de colesterol unido a las lipoproteínas de alta densidad se asocian de manera independiente a enfermedad coronaria aguda en pacientes que ingresan por dolor torácico. Rev Esp Cardiol. 2012;65:319-25.

17. Cordero A, Lopez-Palop R, Bertomeu-González V, Carrillo P, Moreno-Arribas J, Bertomeu-Martínez V. Perfil clínico y pronóstico de los pacientes con síndrome coronario agudo y colesterol unido a lipoproteínas de baja densidad $<70 \mathrm{mg} / \mathrm{dl}$. Rev Esp Cardiol. 2013;66:588-9.

18. Cordero A, Lopez-Palop R, Carrillo P, Miralles B, Masiá M, Bertomeu-Martínez V. Valor pronóstico de la escala INTERHEART-colesterol para pacientes que ingresan por dolor torácico. Rev Esp Cardiol. 2014;67:578-80.

19. Cubedo J, Padro T, Alonso R, Cinca J, Mata P, Badimon L. Differential proteomic distribution of TTR (pre-albumin) forms in serum and HDL of patients with high cardiovascular risk. Atherosclerosis. 2012;222(1):263-9.

20. Garcia-Moll X, Fácila L, Conthe P, Zapata A, Artigas $\mathrm{R}$, Bertomeu V, et al. ¿Cómo se usan los bloqueadores beta en España? Análisis de las limitaciones para su uso en medicina interna y cardiología: estudio CARACTER-BETA. Rev Esp Cardiol. 2011;64:883-90.

21. Jover A, Corbella E, Muñoz A, Millán J, Pintó X, Mangas A, et al. Prevalencia del síndrome metabólico y de sus componentes en pacientes con síndrome coronario agudo. Rev Esp Cardiol. 2011;64:579-86.

22. Machon M, Basterretxea M, Martinez-Camblor $\mathrm{P}$, Aldasoro E, Vicente JM, Larranaga N. Sex differences in relative survival and prognostic factors in patients with a first acute myocardial infarction in guipuzcoa, Spain. Rev Esp Cardiol (Engl Ed). 2010;63(6):649-59.

23. Martinez-Quintana E, Rodriguez-Gonzalez F, Nieto-Lago V, Novoa FJ, Lopez-Rios L, Riano-Ruiz M. Serum glucose and lipid levels in adult congenital heart disease patients. Metabolism. 2010;59(11):1642-8.

24. Millán J, Pedro-Botet J, Muñoz A, Corbella E, Mangas A, Zúñiga M, et al. Perfil lipídico residual en la cardiopatía isquémica recurrente. Med Clin (Barc). 2012;138:238-41.

25. Olivencia PL, Bueno CA, Soto Blanco JM, Yuste Ossorio ME, Barranco RF. Acute coronary syndrome in women. Gender differences. Med Clin (Barc). 2011;137(14):623-30.

26. Pérez De Isla L, Saltijeral Cerezo A, Vitale G, González Timón B, Torres Do Rego A, Álvarez-Sala Walther LA. Prevalencia de colesterol LDL inadecuado en pacientes con enfermedad coronaria y/o diabetes mellitus tipo 2 (Prevalence of inappropriate LDL cholesterol levels in patients with coronary disease and/or type 2 diabetes). Rev Clin Esp. 2012;212(10):475-81.

27. Pineda J, Marin F, Marco P, Roldan V, Valencia J, Ruiz-Nodar JM, et al. The prognostic value of biomarkers after a premature myocardial infarction. Int J Cardiol. 2010;143(3):249-54.

28. Rodríguez-Roca GC, Rodríguez-Padial L, Alonso-Moreno FJ, Romero-Gutiérrez A, Akerström F, Segura-Fragoso A, et al. Perfil lipídico en pacientes ingresados por síndrome coronario agudo en España: datos del área sanitaria de Toledo entre los años 2005 y 2008. Semergen. 2013;39:298-303.

29. Ruiz-Bailén M, Romero-Bermejo FJ, Expósito-Ruiz M, Zamora-Zamora F, Martínez-Ramírez MJ, Castillo-Rivera AM, et al. Tratamiento precoz con estatinas en el infarto agudo de miocardio. Med Intensiv. 2014;38:11-20.

30. Sanfelix-Gimeno G, Peiro $S$, Ferreros I, Perez-Vicente R, Librero J, Catala-Lopez F, et al. Adherence to evidence-based therapies after acute coronary syndrome: a retrospective population-based cohort study linking hospital, outpatient, and pharmacy health information systems in Valencia, Spain. J Manag Care Pharm. 2013;19(3):247-57.

31. Sicras-Mainar A, Fernández De Bobadilla J, Navarro-Artieda R, Martín I, Varela-Moreno C. Morbimortalidad y consumo de recursos asociados tras síndrome coronario agudo en una población española. Rev Clin Esp. 2011;211:560-71. 
32. Lahoz C, Mostaza J, Mantilla M, Taboada M, Tranche S, López-Rodríguez I, et al. Prevalencia de síndrome metabólico en pacientes con enfermedad coronaria estable: objetivos terapéuticos y utilización de fármacos cardiovasculares. Rev Clin Esp. 2011;211:1-8.

33. Lázaro P, Murga N, Aguilar D, Hernández-Presa MA. Inercia terapéutica en el manejo extrahospitalario de la dislipemia en pacientes con cardiopatía isquémica. Estudio Inercia. Rev Esp Cardiol. 2010;63:1428-37.

34. Otero-Raviña F, Mazon-Ramos P, Grigorian-Shamagian L, Nores-Lorenzo A, Zugaza-Gurruchaga L, Seoane-Blanco R, et al. Effect of diabetes on the clinical characteristics and prognosis of patients with chronic ischemic heart disease. The CIBAR study. Rev Esp Cardiol. 2010;63(11):1371-6.

35. Lahoz C, Mostaza JM, Tranche S, Martin-Jadraque R, Mantilla MT, Lopez-Rodriguez I, et al. Atherogenic dyslipidemia in patients with established coronary artery disease. Nutr Metab Cardiovasc Dis. 2012;22(2):103-8.

36. Barrios V, Escobar C, Murga N, Quijano JJ. Clinical profile and management of patients with chronic ischemic heart disease according to age in the population daily attended by cardiologists in Spain: the ELDERCIC study. Eur J Intern Med. 2010;21(3):180-4.

37. González-Juanatey JR, Cordero A, Vitale GC, González-Timón B, Mazón P, Bertomeu V. Magnitud y características del riesgo residual lipídico en pacientes con antecedentes de revascularización coronaria: estudio ICP-Bypass. Rev Esp Cardiol. 2011;64:862-8.

38. Barrios V, Escobar C, Calderon A. Clinical profile and management of patients with hypertension and chronic ischemic heart disease according to BMI. Obesity (Silver Spring). 2010;18(10):2017-22.

39. Vidal-Pérez R, Otero-Raviña F, Domínguez-López J, Fabeiro-Romero D, Gómez-Vázquez JL, de Blas-Abad P, et al. Características clínicas y pronóstico de pacientes con enfermedad cerebrovascular crónica (estudio ICBAR). Rev Neurol. 2011;53:449-56.

40. Abellán Alemán J, Ruilope Urioste LM, Leal Hernández M, Armario García P, Tiberio López G, Martell Claros N. Control de los factores de riesgo cardiovascular en pacientes con ictus atendidos en Atención Primaria en España. Estudio ICTUSCARE. Med Clin (Barc). 2011;136:329-35.

41. Rubio Gil E, Martínez Pastor A, López-Picazo Ferrer J, Leal Hernández M, Morales Ortiz A, Martinez
Navarro A, et al. Calidad del registro en OMI-AP de los pacientes con ictus seguidos en atención primaria quality of records in OMI-AP of the patients with stroke followed in primary care. Rev Calid Asist. 2010;25(6):341-7.

42. Mostaza JM, Puras E, Blasco M, Lahoz C, Samaniego ML. Utilización de tratamientos cardiovasculares preventivos y consecución de objetivos terapéuticos en pacientes con enfermedad arterial periférica. Rev Esp Cardiol. 2012;65:713-8.

43. Félix-Redondo FJ, Fernández-Berges D, Grau M, Baena-Diez JM, Mostaza JM, Vila J. Prevalence and clinical characteristics of peripheral arterial disease in the study population Hermex. Rev Esp Cardiol (Engl Ed). 2012;65(8):726-33.

44. Dalli E, Colomer E, Tormos MC, Cosin-Sales J, Milara J, Esteban E, et al. Crataegus laevigata decreases neutrophil elastase and has hypolipidemic effect: a randomized, double-blind, placebo-controlled trial. Phytomedicine. 2011;18(8-9):769-75.

45. Piulats-Egea N, Albaigès-Ràfols MC, Solé-Sancho J, Benito-Badorrey MB, Casas-Rodríguez J, Sendra-Pons M. Cardiopatía isquémica en población inmigrante. Hipertens Riesgo Vasc. 2014;31:40-4.

46. Ruescas-Escolano E, Orozco-Beltran D, Gaubert-Tortosa M, Navarro-Palazón A, Cordero-Fort A, Navarro-Pérez J, et al. El estudio PROPRESE: resultados de un nuevo modelo organizativo en atención primaria para pacientes con cardiopatía isquémica crónica basado en una intervención multifactorial. Aten Primaria. 2014;46:10-5.

47. Arrospide A, Mar J, Vivancos-Mora J, Rejas-Gutierrez J, Caro J. Cost-effectiveness analysis of using high doses of atorvastatin for the secondary stroke prevention in Spain. Rev Neurol. 2010;51(1):1-11.

48. Brea D, Roquer J, Serena J, Segura T, Castillo J. Oxidative stress markers are associated to vascular recurrence in non-cardioembolic stroke patients non-treated with statins. BMC Neurol. 2012;12:65.

49. Clua-Espuny JL, Piñol-Moreso JL, Gil-Guillén VF, Orozco-Beltrán D, Panisello-Tafalla A, Lucas-Noll J, et al. Resultados de prevención cardiovascular primaria y secundaria en pacientes con ictus: riesgo de recurrencia y supervivencia asociada (estudio Ebrictus). Rev Neurol. 2012;54:81-92.

50. Jiménez-Caballero PE, López-Espuela $\mathrm{F}$, Portilla-Cuenca JC, Jiménez-Gracia MA, Casado-Naranjo I. Detección de factores de riesgo vascular y fibrilación auricular no conocida en pacientes ingresados en la unidad de ictus. Rev Neurol. 2013;56:464-70. 
51. López-Farre AJ, Zamorano-León JJ, Segura A, Mateos-Caceres PJ, Modrego J, Rodriguez-Sierra P, et al. Plasma desmoplakin I biomarker of vascular recurrence after ischemic stroke. J Neurochem. 2012;121(2):314-25.

52. Martinez-Sanchez $P, \quad$ Fuentes B, Fernandez-Dominguez J, Ortega-Casarrubios ML, Aguilar-Amar MJ, Abenza-Abildua MJ, et al. Young women have poorer outcomes than men after stroke. Cerebrovasc Dis. 2011;31(5):455-63.

53. Merida-Rodrigo L, Poveda-Gomez F, Camafort-Babkowski M, Rivas-Ruiz F, Martin-Escalante MD, Quiros-Lopez R, et al. Long-term survival of ischemic stroke. Rev Clin Esp. 2012;212(5):223-8.

54. Pardo-Cabello AJ, Bermudo-Conde S, Manzano-Gamero V, Gómez-Jiménez FJ, Torres-Puchol JdlH. Implementación de las guías de práctica clínica sobre ictus isquémico agudo en atención especializada. Implementation of clinical practice guidelines for acute ischaemic stroke in specialist. Neurologia. 2013;28(3):137-44.

55. Purroy F, Montserrat J, Begue R, Gil MI, Quilez A, Sanahuja J, et al. Higher carotid intima media thickness predicts extracranial vascular events and not stroke recurrence among transient ischemic attack patients. Int J Stroke. 2012;7(2):125-32.

56. Rodriguez-Sanz A, Fuentes B, Martinez-Sanchez P, Prefasi D, Martinez-Martinez M, Correas E, et al. High-density lipoprotein: a novel marker for risk of in-hospital infection in acute ischemic stroke patients? Cerebrovasc Dis. 2013;35(3):291-7.

57. Roquer J, Rodriguez-Campello A, Cuadrado-Godia E, Giralt-Steinhauer E, Jimenez-Conde J, Degano IR, et al. Ischemic stroke in prediabetic patients. J Neurol. 2014;261:1866-70.

58. Rubio Gil E, Martínez Pastor A, López-Picazo Ferrer J, Leal Hernández M, Martínez Navarro A, Abellán Alemán J. Estudio de los registros de seguimiento y control de los factores de riesgo en pacientes con ictus seguidos en atención primaria por historia clínica informatizada (OMI-AP). Estudio ictus-OMI. Aten Primaria. 2011;43:209-10.

59. Ruiz-Ares G, Fuentes B, Martinez-Sanchez P, Martinez-Martinez M, Diez-Tejedor E. Utility of the assessment of echogenicity in the identification of symptomatic carotid artery atheroma plaques in ischemic stroke patients. Cerebrovasc Dis. 2011;32(6):535-41.

60. Serrano-Villar S, Fresco G, Ruiz-Artacho P, Bravo A, Valencia C, Fuentes-Ferrer $M$, et al. Stroke in the very old: myths and realities. Med Clin (Barc). 2013;140(2):53-8.
61. Polo-Romero F, Santisteban-López Y, Ramírez-Relinque L, Rodríguez-Martín B. Estrategias para mejorar el control lipídico: aumentar dosis de estatinas o asociar ezetimiba? Rev Clin Esp. 2010;210:425-6.

62. Estirado E, Lahoz C, Laguna F, Garcia-Iglesias F, Gonzalez-Alegre MT, Mostaza JM. Metabolic syndrome in patients with peripheral arterial disease. Rev Clin Esp. 2014;214:437-44.

63. Ferreras-Amez JM, Sanjuán-Domingo R, Visiedo-Sánchez S, Sarrat-Torres MA, Vicente-Molinero A, Abadía-Gallego $\mathrm{V}$, et al. Análisis del control de factores de riesgo cardiovascular en pacientes con enfermedad arterial periférica. Semergen. 2013;39:63-9.

64. Félix-Redondo FJ, Fernández-Bergés D, Palomo-Cobos L, Buitrago-Ramírez F, Pérez-Castán JF, Lozano-Mera L. Prevalencia de consumo de alcohol y factores de riesgo cardiovascular en un área sanitaria de Extremadura. Estudio Hermex. Aten Primaria. 2012;44:201-8.

65. Martínez-Hervás J, Priego A, Lorente R, Molina M, Navarro-Hidalgo MI, Real JT, et al. Arteriosclerosis carotídea subclínica en pacientes con hiperlipidemia familiar combinada. Evolución tras dos años de tratamiento con dosis altas de atorvastatina. Med Clin (Barc). 2012;138:1-6.

66. Mazon-Ramos P, Cordero A, Gonzalez-Juanatey JR, Bertomeu MV, Delgado E, Vitale G, et al. Control of cardiovascular risk factors in revascularized patients with diabetes: a subanalysis of the ICP-Bypass Study. Rev Esp Cardiol (Engl Ed). 2015;68:115-20.

67. Pérez A, González-Blanco C, Hernández-Presa MÁ, Chaves J. Estrategia de tratamiento de la dislipemia y consecución de objetivos en la población española con diabetes tipo 2 sin enfermedad cardiovascular. Endocrinol Nutr. 2011;58:283-90.

68. Roca-Rodríguez MM, Carral-San Laureano F, Baena-Nieto G, Aguilar-Diosdado M. Evaluación del grado de consecución de objetivos de control metabólico en pacientes con diabetes mellitus tipo 2. Endocrinol Nutr. 2010;57:434-439.

69. López-Simarro F, Brotons C, Moral I, Cols-Sagarra C, Selva A, Aguado-Jodar A, et al. Inercia y cumplimiento terapéutico en pacientes con diabetes mellitus tipo 2 en atención primaria. Med Clin (Barc). 2012;138:377-84.

70. Pérez De Isla L, Saltijeral Cerezo A, Vitale G, González-Timón B, Torres Do Rego A, Álvarez-Sala Walther LA. Cifras de colesterol adecuadas en pacientes coronarios y diabéticos. Análisis según especialidades médicas y comunidades autónomas. Rev Esp Cardiol. 2013;66:748-9. 
71. Millan J, Alegría E, Guijarro C, Lozano JV, Vitale GC, González-Timón B, et al. Dislipemia en población diabética tratada con estatinas. Resultados del estudio DYSIS en España. Med Clin (Barc). 2013;141:430-6.

72. Pedro-Botet J, Mostaza JM, Pinto X, Banegas JR. Consecución del objetivo terapéutico del colesterol de las lipoproteínas de baja densidad en las unidades de lípidos y riesgo vascular de la Sociedad Española de Arteriosclerosis. Clin Investig Arterioscler. 2013;25:155-63.

73. González-Juanatey JR, Millán J, Alegría E, Guijarro C, Lozano JV, Vitale GC. Prevalencia y características de la dislipemia en pacientes en prevención primaria y secundaria tratados con estatinas en España. Estudio DYSIS-España. Rev Esp Cardiol. 2011;64:286-94.

74. Martinez-Hervas S, Carmena R, Ascaso J, Real J, Masana L, Catala M, et al. Prevalence of plasma lipid abnormalities and its association with glucose metabolism in Spain: the di@bet.es study. Clin Investig Arterioscler. 2014;26(3):107-14.

75. De la Peña A, Roca-Cusachs À, Suarez C, Bonet S. Riesgo vascular en las consultas de Medicina Interna. Estudio MICARE. Med Clin (Barc). 2013;140:246-54.

76. Divisón-Garrote JA, Massó-Orozco J, Carrión-Valero L, López-Abril J, Carbayo-Herencia JA, Artigao-Rodenas LM, et al. Evolución de la prevalencia de factores de riesgo y del riesgo cardiovascular global en población mayor de 18 años de la provincia de Albacete (1992-94 a 2004-06). Rev Esp Salud Publica. 2011;85:277-86.

77. Brotons C, Moral I, Soriano N, Cuixart L, Osorio D, Bottaro D, et al. Impacto de la utilización de las diferentes tablas SCORE en el cálculo del riesgo cardiovascular. Rev Esp Cardiol. 2014;67:94-100.

78. Félix-Redondo FJ, Fernández-Bergés D, Fernando-Pérez J, Zaro MJ, García A, Lozano L, et al. Prevalencia, detección, tratamiento y grado de control de los factores de riesgo cardiovascular en la población de Extremadura (España). Estudio HERMEX. Aten Primaria. 2011;43:426-34.

79. Zamora A, de Fernandez BF, Carrion C, Vazquez G, Paluzie G, Elosua R, et al. Pilot study to validate a computer-based clinical decision support system for dyslipidemia treatment (HTE-DLP). Atherosclerosis. 2013;231(2):401-4.

80. Suades R, Padro T, Alonso R, Mata P, Badimon L. Lipid-lowering therapy with statins reduces microparticle shedding from endothelium, platelets and inflammatory cells. Thromb Haemost. 2013;110(2):366-77.

81. Antón-García F, Correcher-Salvador E, Rodríguez-Lagos FA, González-Caminero S. Evaluación durante 6 años de la dislipidemia en un centro de salud. Importancia de las acciones de mejora. Semergen. 2014;40:241-6.

82. Díaz-Díaz J, Suárez-Tembra M, Díaz-Peromingo J, Pena-Seijo M, Martínez-Ramonde T, Pose-Reino A. Eficacia y seguridad de ezetimiba en unidades de riesgo vascular. Rev Clin Esp. 2011;211:283-90.

83. Taylor F, Huffman MD, Macedo AF, Moore TH, Burke M, Davey SG, et al. Statins for the primary prevention of cardiovascular disease. Cochrane Database Syst Rev. 2013;1:CD004816.

84. Huerta JM, Tormo MJ, Gavrila D, Navarro C. Cardiovascular risk estimated after 13 years of follow-up in a low-incidence Mediterranean region with high-prevalence of cardiovascular risk factors. BMC Public Health. 2010;10:640.

85. Marin A, Medrano MJ, Gonzalez J, Pintado $\mathrm{H}$, Compaired V, Barcena M, et al. Risk of ischaemic heart disease and acute myocardial infarction in a Spanish population: observational prospective study in a primary-care setting. BMC Public Health. 2006;6:38.

86. Ministerio de Sanidad Servicios Sociales e Igualdad, Agencia Española de Medicamentos y Productos Sanitarios. Utilización de medicamentos hipolipemiantes en España durante el periodo 2000-2012. Madrid: MSSSI; 2014. Informe de utilización de medicamentos: U/HLP/V1/17012014.

87. Mindell J, Aresu M, Zaninotto P, Falaschetti E, Poulter N. Improving lipid profiles and increasing use of lipid-lowering therapy in England: results from a national cross-sectional survey-2006. Clin Endocrinol (Oxf). 2011;75(5):621-7.

88. Omboni S, Carabelli G, Ghirardi E, Carugo S. Awareness, treatment, and control of major cardiovascular risk factors in a small-scale Italian community: results of a screening campaign. Vasc Health Risk Manag. 2013;9:177-85.

89. Verhave JC, Troyanov S, Mongeau F, Fradette L, Bouchard J, Awadalla P, et al. Prevalence, awareness, and management of CKD and cardiovascular risk factors in publicly funded health care. Clin J Am Soc Nephrol. 2014;9(4):713-9. 